\title{
B ultrasound-guided hyperthermic intraperitoneal perfusion chemotherapy for the treatment of malignant ascites
}

\author{
SHUZHONG CUI ${ }^{1}$, MINGCHEN BA ${ }^{2}$, YUNQIANG TANG ${ }^{2}$, JIFANG LIU $^{2}$, YINBING WU $^{2}$, \\ BIN WANG $^{2}$, XIANGLIANG ZHANG ${ }^{2}$, HONGSHENG TANG $^{2}$ and SHIZHEN ZHONG ${ }^{1}$ \\ ${ }^{1}$ The Institute of Anatomy, School of Basic Medical Sciences, Southern Medical University, Guangzhou 510515; \\ ${ }^{2}$ Intracelom Hyperthermic Perfusion Therapy Center, Cancer Hospital of \\ Guangzhou Medical College, Guangzhou 510095, P.R. China
}

Received January 12, 2012; Accepted March 6, 2012

DOI: $10.3892 / o r .2012 .1913$

\begin{abstract}
To minimize invasive surgery, we employed B ultrasound to guide the placement of the catheters used in continuous circulatory hyperthermic intraperitoneal perfusion chemotherapy (CHIPC) in malignant ascites treatment. Thirty-two patients with malignant ascites were treated with CHIPC guided by B-mode ultrasound. Ascites were originally from ovarian cancer (11 cases), gastric cancer (10 cases), colorectal cancer (9 cases) and pancreatic cancer ( 2 cases). The CHIPC was carried out at $43^{\circ} \mathrm{C}$ for 90 min with $0.9 \%$ saline solution as a carrier containing cisplatin and doxorubicin or mitomycin-C as therapeutic reagents depending on the type of the primary tumor. The therapeutic efficacy, postoperative complications and survival period of these patients were assessed with follow-up examinations. Among all participates to be assessed with ascites, 26 and 4 patients showed complete remission (CR) and partial remission (PR) respectively, with an objective remission rate (ORR) of $93.75 \%$. The KPS scores were elevated by $23.1 \pm 9.0$ after 3 sessions of ultrasound guided CHIPC and the quality of life (QOF) of patients was significantly improved $(\mathrm{p}<0.01)$. The median survival time was 9 months and 18 patients survived between 3 and 30 months after CHIPC treatment. Additionally, patients with different types of cancers significantly differed in the survival time $(\mathrm{p}<0.01)$. A novel approach of using B ultrasound guided CHIPC for the treatment of malignant ascites demonstrated satisfactory outcomes. The approach shows benefit in minimizing invasive surgery, improving the patient QOF and prolonging survival time.
\end{abstract}

Correspondence to: Dr Mingchen Ba, Intracelom Hyperthermic Perfusion Therapy Center, Cancer Hospital of Guangzhou Medical College, Guangzhou 510095, P.R. China

E-mail: bamingchen2011@126.com

Dr Shizhen Zhong, The Institute of Anatomy, School of Basic Medical Sciences, Southern Medical University, Guangzhou 510515, P.R. China

E-mail: shizhenzhong@126.com

Key words: B ultrasound, hyperthermic intraperitoneal perfusion, chemotherapy, malignant ascites, peritoneal carcinomatosis

\section{Introduction}

Malignant ascites frequently occur in patients with ovarian, uterine, gastrointestinal tract (stomach and intestines), breast and pancreatic cancers, in which fluid containing cancer cells accumulate in the abdomen cavity. Without treatment, those patients would have a poor prognosis with a survival period of less than 2 months (1). Invasive therapy (e.g., paracentesis) is efficient for attenuation of the symptom, but reiterating paracentesis may cause biochemical disturbances in vivo with the associated complications. A more effective therapy which could reduce ascites with lesser complications seems necessary for improving quality of life (QOF) of the patients and prolonged survival of patients $(2,3)$.

Continuous circulatory hyperthermic intraperitoneal perfusion chemotherapy (CHIPC) is a novel adjuvant therapy for peritoneal carcinomatosis with a mechanical scavenging effect on peritoneal suspension cancer cells (4-8). Warm chemotherapy liquid containing high-doses of anti-cancer drugs is administered into peritoneal cavity to react with tumor metastases. It has benefits of lessen drugs entering circulation and systemic toxic side effects, and a greater cytotoxic effect of hyperthermia on cancer (9-12).

A great clinical efficacy on the treatment of malignant ascites using CHIPC has been demonstrated (13-17). During traditional CHIPC, the perfusion catheter is traditionally placed through an invasive laparotomy which requires general anesthesia or epidural anesthesia (18-23). Although the procedure has been improved recently with the development of minimally invasive surgical techniques (MIS) (1,13-16), the laparoscope-assisted CHIPC requires special laparoscope devices (usually expensive) with expertise in the field, which limits its development and clinical application. In addition, many laparoscopic surgeons may also have a difficulty in prevention and treatment of seeding/ metastasis of the punctured hole(s) on abdominal wall $(13,14,17)$.

$\mathrm{B}$ ultrasound is non-invasive, less expensive, repeatable in use, and has a high specificity in the diagnosis for ascites and liquid estimation in the peritoneal cavity. It also has been widely used by clinicians for guiding intraperitoneal punctuation $(24,25)$ and intraperitoneal drainage construction $(26,27)$. Therefore, it seems rational to replace laparoscope with B ultrasound during the procedure of CHIPC. We have, 
for the first time, introduced B ultrasound to guide CHIPC for the treatment of malignant ascites induced by solid cancers. The clinical efficacy, side effects and prognosis of our improved CHIPC procedure were investigated.

\section{Materials and methods}

Inclusion criteria and clinical data. Thirty-two ovarian cancer (OC), gastric cancer (GC), colorectal cancer (CRC) and pancreatic cancer patients complicated with malignant ascites patients (Table I) administered in our hospital from 2007 to 2011 were included in this study. The ascites volumes ranged from 4000 to $9000 \mathrm{ml}$ estimated by B volume of intraperitoneal drainage and the Karnofsky performance scale (KPS) of QOF scored 40-70. The origin tumors of these patients were diagnosed and confirmed by laparotomy exploration and/or graphology or fiber endoscope examination and/or serum tumor biomarker examination (CA125, CEA and CA199) or ascites cytology examination. The existence and size of ascites were examined by B ultrasound and/or computed tomography (CT) scan. Diagnosis of ascites related to unresectable carcinomatosis was made preoperatively in all cases by standard clinical and radiological assessments (cytology and CT scan). Peritoneal carcinomatosis was confirmed by PET-CT and/or other graphology examination or detection of peritoneal suspension tumor cells. Patients with extensive abdomen surgical procedures and/or with insignificant ascites were excluded from this study. Prior to the therapy, all participates were informed on the palliative role of the procedure, any risks may be involved, possible complications and expected benefits, and then consents were obtained as clinical research guidelines. This study was approved by Medical Ethics Committee of Cancer Hospital of Guangzhou Medical College.

$B$ ultrasound-guided placement of catheters for CHIPC. In a standard operating theater, patients were placed in a supine position. Pethidine hydrochloride $(75 \mathrm{mg}$ ) and promethazine hydrochloride $(25 \mathrm{mg})$ were administered by intramuscular injection prior to placing CHIPC catheters. Propofol, as an anesthetic agent, was given intravenously via a continuous vein pump with dose (3-8 $\mathrm{ml} / \mathrm{h}$ ) adjusted according to patient status. B ultrasound examinations on all 4 abdominal quadrants were performed to choose puncture point which should be in the region of a larger ascites together with no adhesion between abdominal wall and the tissues of peritoneal cavity without original abdominal incision or tumor. A $1.2-\mathrm{cm}$ incision was made by a Hasson trocar $(1.2 \mathrm{~cm}$ in diameter) at the punctuation point after administering $0.5 \%$ lidocaine (anesthetic agent) locally before the infusion and outflow catheters with multiple side holes (inner diameter $0.8 \mathrm{~cm}$, diameter $1.0 \mathrm{~cm}, 100 \mathrm{~cm}$ in length) were placed into intraperitoneal cavity. The infusion catheters were sited in the left and right upper quadrants of the intraperitoneal cavity with an inside length of $40-80 \mathrm{~cm}$, and the outflow catheters were placed in the pelvic cavity of the left and right lower quadrants with a same length as that of the infusion catheters. All port sites were fixed to abdominal wall by cutaneous sutures as shown in Fig. 1 .

CHIPC procedures. CHIPC was performed by our selfdeveloped 'BR-TRG-II type high-precision hyperthermic
Table I. Characteristics of patients undergoing B-mode ultrasound guided CHIPC $(n=32)$.

$\mathrm{n}(\%) /$ mean $\pm \mathrm{SD}$

\begin{tabular}{ll}
$\begin{array}{l}\text { Gender } \\
\text { Male }\end{array}$ & $13(41)$ \\
Female & $19(59)$ \\
Age (years) & $59.41 \pm 10.54$ \\
$\begin{array}{l}\text { Cancer types and treatment before CHIPC } \\
\text { Ovarian cancer }\end{array}$ & $5(15.63)$ \\
$\begin{array}{l}\text { Peritoneal carcinomatosis post ovarian } \\
\text { cancer cytoreductive surgery }\end{array}$ & $6(18.75)$ \\
$\begin{array}{l}\text { Gastric cancer } \\
\text { Peritoneal carcinomatosis post gastric }\end{array}$ & $4(18.75)$ \\
cancer resection & $4(12.5)$ \\
$\begin{array}{l}\text { Colorectal cancer } \\
\text { Peritoneal carcinomatosis post colorectal } \\
\text { colorectal cancer resection }\end{array}$ & $3(9.38)$ \\
Pancreatic cancer & $6(18.75)$ \\
$\begin{array}{l}\text { Disease courses (days) } \\
\text { Ascite volume (ml) }\end{array}$ & $2(6.25)$ \\
Cases of free cancer cells in ascites & $18.91 \pm 10.57$ \\
\hline
\end{tabular}

intraperitoneal perfusion treatment system' with a precise of $\pm 0.15^{\circ} \mathrm{C}$ on temperature control and of $\pm 5 \%$ on flow control, which couples with an automatic cooling function. The devices, the only ones of this kind, have been approved by State Food Drug Administration Firearms (SFDA) of China (approval no. 2009-3260924) (Fig. 2).

Our CHIPC therapy consisted of three sessions, the first was completed in the operating room in original status of anesthesia post-placement of catheters/infusion tubes guided by B-ultrasound on the same day, and the second and third sessions were followed on in the intensive care unit (ICU) on the first and the following days, respectively. Saline solution ( $0.9 \%$ ) (equivalent to the volume of cavity, i.e., $4500-6000 \mathrm{ml}$ ) was added into the tailor-made infusion bag and delivered via infusion tubes over $90 \mathrm{~min}$ with a velocity of 450-600 $\mathrm{ml} / \mathrm{min}$ and an inflow temperature of $43^{\circ} \mathrm{C}$ in attempt to achieve an interior abdominal temperature of $41.5-42.5^{\circ} \mathrm{C}$. The hyperthermic intraperitoneal chemotherapeutic agents spiked in the perfusion fluid were: i) cisplatin $\left(50 \mathrm{mg} / \mathrm{m}^{2}\right.$ of body surface) and doxorubicin $(50 \mathrm{mg} /$ $\mathrm{m}^{2}$ of body surface), and ii) mitomycin-C $\left(12.5 \mathrm{mg} / \mathrm{m}^{2}\right.$ of body surface) for the ascites originated respectively from ovarian cancer, and from rectal colon or gastric or pancreatic cancer, which were used equally in all 3 sessions of CHIPC. Once the third session had been completed, the peritoneal perfusion liquid and ascites were drained out. Two infusion and one outflow catheters were then pulled out, keeping one outflow catheter as a drainage catheter for 1-3 days. 


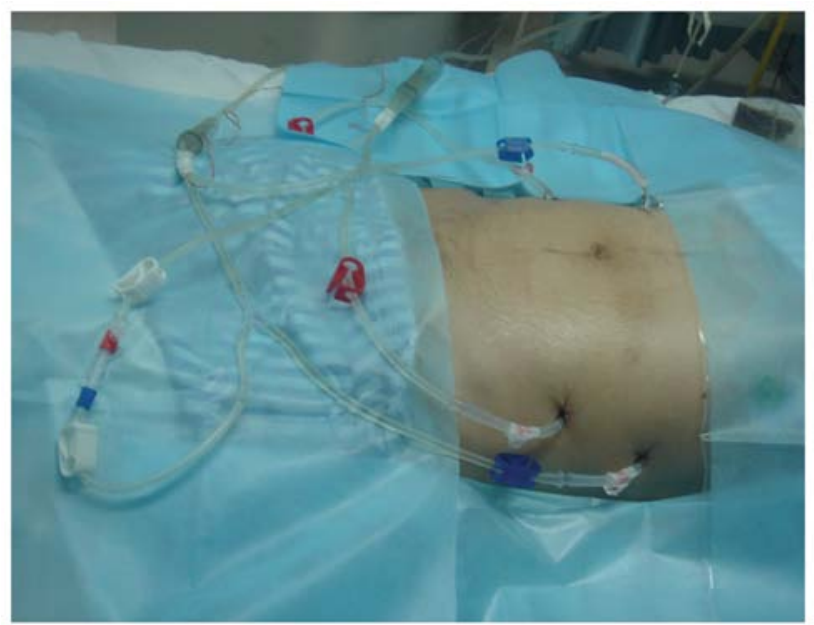

Figure 1. The placement sites of the infusion and outflow catheters in CHIPC guided by B ultrasound. Red clips mark two infusion catheters, blue clips mark two outflow catheters, and white clips mark loop circuit for CHIPC preparation.

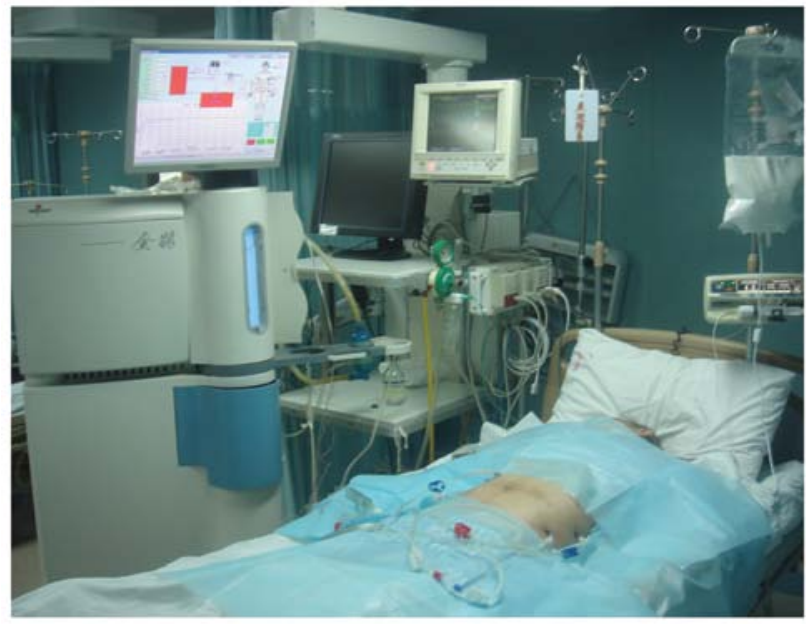

Figure 2. The BR-TRG-II type high precision hyperthermic perfusion intraperitoneal treatment system used in this study.
Evaluation and determination of efficacy. B ultrasonic and/or CT examinations were performed at least fortnightly to assess the therapeutic efficacy on ascites status/remission and tumor progression. KPS scores for QOL of all participates prior to and after 2 weeks post-CHIPC procedure were used for the evaluation. Clinical efficacy on all participates was classified into three grades according to our previous modified WHO criteria on efficacy assessment in malignant tumors (13): i) complete remission (CR): ascites are completely absorbed after treatment sustained over 4 weeks; ii) partial remission (PR): ascites are reduced by $50 \%$; this is sustained over 4 weeks; iii) no consequence (NC): ascites are not reduced obviously or increased after treatment.

Statistical analysis. Overall survival and survival in OC, GC and CRC groups were analyzed and compared by the Kaplan-Meier method with the use of GraphPad Prism software, version 5.01 (GraphPad, San Diego, CA, USA). Differences in survival were determined by the log-rank test for statistical significance. $\mathrm{P}<0.05$ was considered statistically significant. Additional data analysis for KPS scores was done by Student's t-test for paired data.

\section{Results}

Clinical efficacy. All 32 participated patients were successfully administered CHIPC guided by B ultrasound with an average time of $35 \mathrm{~min}$ for placement of catheters. The average KPS scores were significantly elevated by $40 \%$ from 54.06 before treatment to 77.19 after CHIPC $(\mathrm{p}<0.001)$. Clinical CR of ascites was achieved in 26 out of 32 patients $(81.25 \%)$, PR was achieved in 4 patients $(12.5 \%)$, and no consequence was observed in 2 patients $(6.25 \%)$. Thus the total objective remission rate (ORR, ORR $=\mathrm{CR}+\mathrm{PR}$ ) of this study was $93.75 \%$, which demonstrated a significant achievement on clinical efficacy with our modified CHIPC (Tables II and III).

Side effect. During the CHIPC, the participating patients showed no significant variation in vital signs or discomforts

Table II. Clinical efficacy of patients with malignant ascites secondary to peritoneal carcinomatosis undergoing CHIPC guided by B ultrasound.

\begin{tabular}{|c|c|c|c|c|}
\hline & Overall & OC patients & GC patients & CRC patients \\
\hline \multicolumn{5}{|l|}{ KPS mark } \\
\hline Before CHIPC & $54.06 \pm 9.46$ & $59.09 \pm 9.44$ & $49 \pm 7.38$ & $52.22 \pm 9.72$ \\
\hline After CHIPC & $77.19 \pm 10.23$ & $82.73 \pm 7.86$ & $72 \pm 11.35$ & $74.44 \pm 8.82$ \\
\hline p-values & $<0.01$ & $<0.01$ & $<0.01$ & $<0.01$ \\
\hline \multicolumn{5}{|l|}{ Therapeutic outcome, n (\%) } \\
\hline $\mathrm{CR}$ & $26(81.25 \%)$ & $11(100 \%)$ & $6(60 \%)$ & $7(77.78 \%)$ \\
\hline PR & $4(12.5 \%)$ & - & $3(30 \%)$ & $1(11.11 \%)$ \\
\hline $\mathrm{NC}$ & $2(6.25 \%)$ & - & $1(10 \%)$ & $1(11.11 \%)$ \\
\hline Median survival (months) & 9 & 13 & 5.5 & 8 \\
\hline
\end{tabular}

CR, complete remission; PR, partial remission; NC. no consequence; OC, ovarian cancer; GC, gastric cancer; CRC, colorectal cancer. 
Table III. Comparison of clinical efficacy of patients with malignant ascites treated by B ultrasound-guided and laparoscopically-assisted CHIPC.

\begin{tabular}{lccc}
\hline & $\begin{array}{l}\text { Clinical } \\
\text { efficacy } \\
\text { (CR+PR) }\end{array}$ & $\begin{array}{c}\text { Median } \\
\text { survival time } \\
\text { (months) }\end{array}$ & $\begin{array}{c}\text { Metastasis } \\
\text { of puncture } \\
\text { hole (\%) }\end{array}$ \\
\hline $\begin{array}{l}\text { B ultrasound- } \\
\text { guided (n=32) }\end{array}$ & $93.75 \%$ & 9 & $18.75 \%$ \\
$\begin{array}{l}\text { Laparoscopically- } \\
\text { assisted (n=33) } \\
\text { p-values }\end{array}$ & $93.90 \%$ & 8 & $18.18 \%$ \\
\hline
\end{tabular}

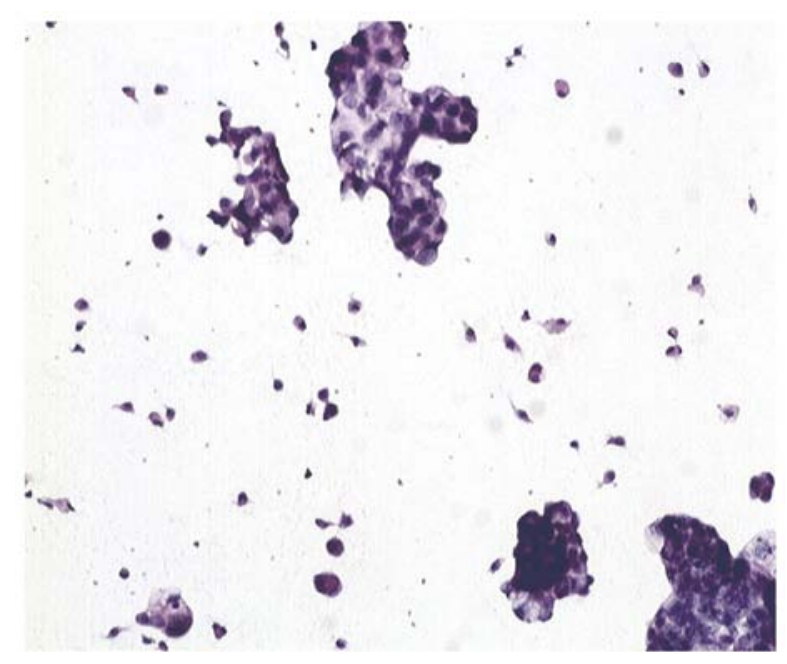

Figure 3. Suspension cancer cells were found in ascites prior to CHIPC (H\&E staining, $\mathrm{x} 100)$.

except transient fever, abdominal distension, and/or abdominal pain. There were 7 cases of first to second degree of bone marrow suppression and 3 cases of mild gastrointestinal reaction. All symptoms were alleviated after the treatment. No intraperitoneal infection or adhesive intestinal obstruction or other complications occurred due to the procedure. Therefore, B-mode ultrasound guided CHIPC can serve as a safe paradigm for malignant ascites.

Follow-up and prognosis. The time periods of follow-up after CHIPC in this study ranged from 3 to 30 months with survival periods ranging from 2 to 30 months (a median survival period of 9 months). Eleven patients with ovarian cancer and peritoneal carcinomatosis post-ovarian cancer cytoreductive surgery have survival time from 7 to 30 months with a median survived time of 13 months; 10 patients with gastric cancer and peritoneal carcinomatosis post-gastric cancer resection have survival time from 2 to 16 months with a median survived time of 5.5 months; and 9 patients with colorectal cancer and peritoneal carcinomatosis post colorectal cancer resection have survival time ranged 5 to 17 months with a median survival time of 8 months (Table II). Furthermore,

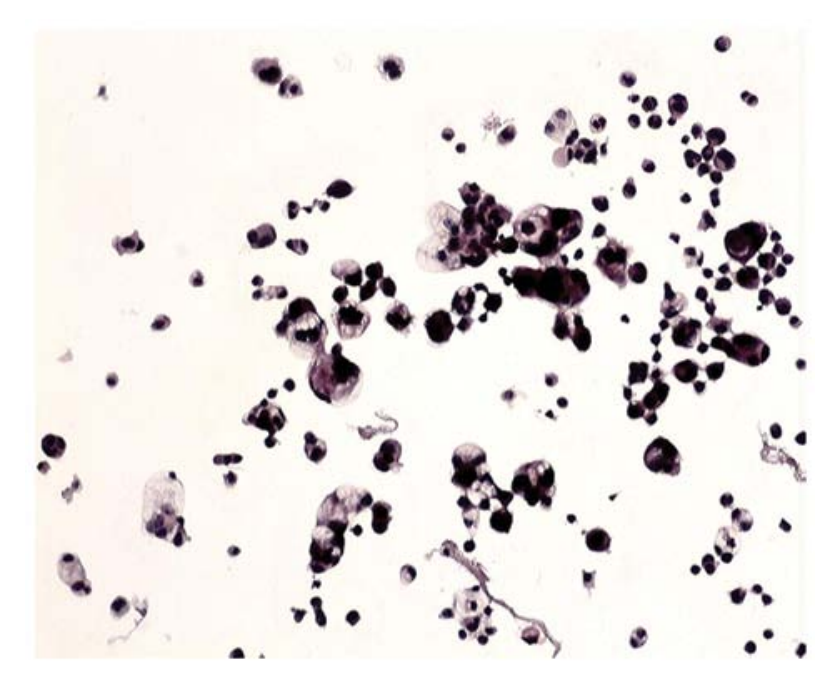

Figure 4. Suspension cancer cells were found in ascites after CHIPC (H\&E staining, x100).

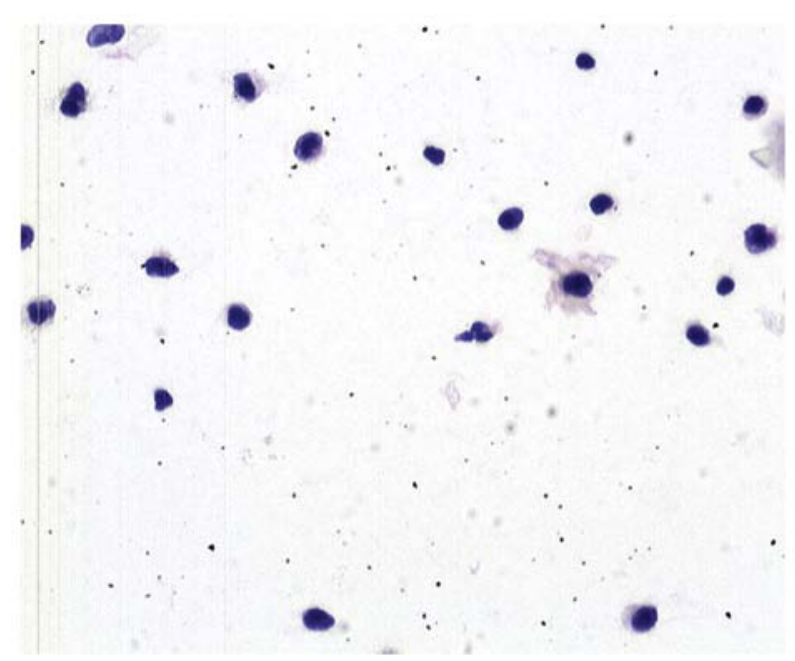

Figure 5. Degenerated and necrotized cells were found in ascites after second CHIPC (H\&E staining, x100).

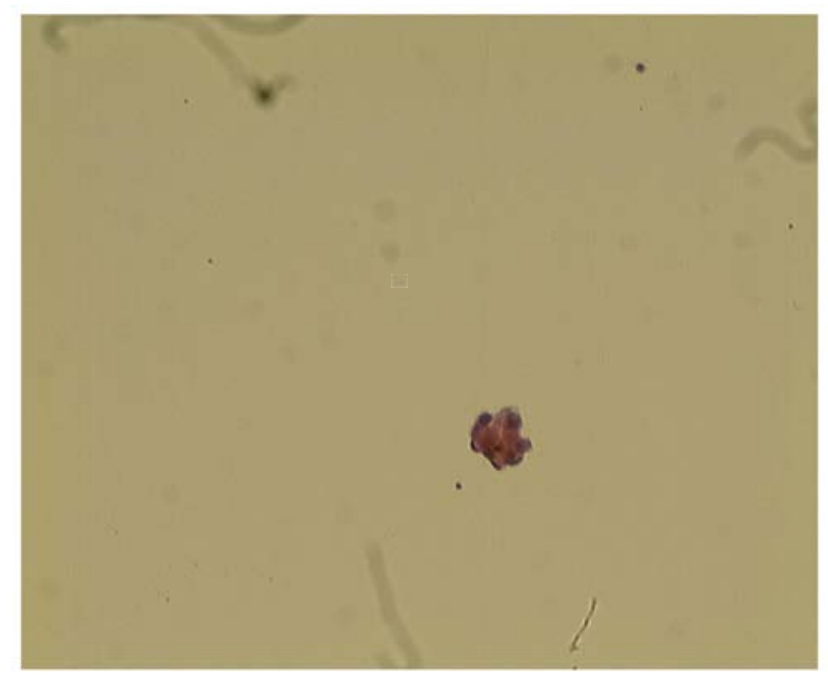

Figure 6. No suspension cancer cells were found in ascites after third CHIPC (H\&E staining, x100). 


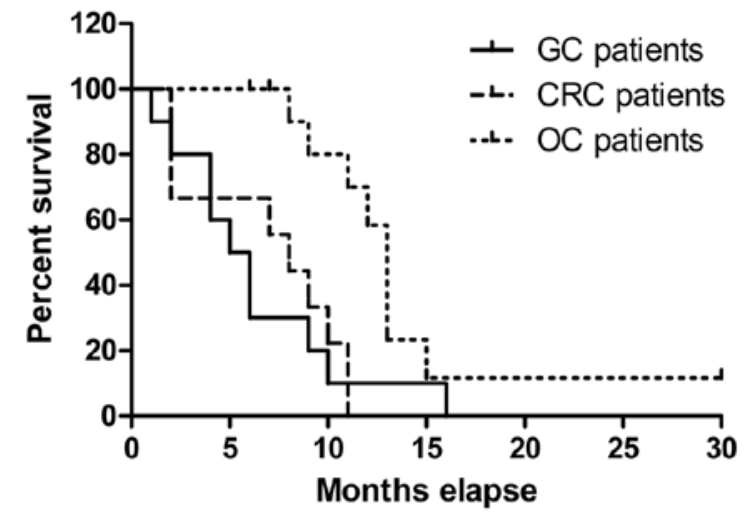

Figure 7. Survival curves of patients with different types of cancers after ultrasound guided CHIPC.

there was a significant difference in survival time of patients with different types of cancers ( $\mathrm{p}<0.01)$ (Fig. 7). In addition, CT scans showed a small fluid accumulation in the omental bursa and in the pelvis in 2 and 1 patients, respectively.

Case study: a 67-year-old woman with $4500 \mathrm{ml}$ ascites containing a great number of free cancer cells (Fig. 3) had a serum CA125 result of $530 \mathrm{IU} / \mathrm{ml}$ without peritoneal mass determined by image examinations. She was diagnosed with ovarian cancer with normal size ovary and malignant ascites. After 3 sessions of CHIPC, free cancer cells in her ascites progressively disappeared and were replaced with necrosis (Figs. 4-6) and serum CA125 values returned to normal four weeks post CHIPC. After receiving two circles systemic chemotherapy post
CHIPC 35 days, she was performed exploratory laparotomy. Tumor mass and tumor metastasis were found in her bilateral ovary and uterus, but not in other organs. Biopsy tissue showed her right ovarian tumor to be an ovarian cancer and a total hysterectomy, bilateral adnexectomy and greater omentectomy were performed. After surgery, she has alive for over 30 months in good health.

\section{Discussion}

Since the 1980s, CHIPC therapeutic approach in the treatment of peritoneal metastatic carcinoma has been applied in the treatments of peritoneal carcinomatosis originated from gastric, colorectal and ovarian cancers, and pseudomyxoma peritonei world widely in challenging difficulties of the therapy regime $(2,3)$. It has achieved satisfactory therapeutic effects $(13,15,18,19)$.

The tradition CHIPC used a laparoscope or perform open operation to assist the placement of catheters, which could result in large traumatic wounds and required costly instruments and expertise. We have, for the first time, introduced B ultrasound in CHIPC to guide the placement of catheters in abdominal wall in the treatment of malignant ascites. This approach largely avoids injury of visceral organs caused by using laparoscopy and the associated complications, in addition to its relatively low cost in operation and less technical and clinical skills required. It also has a greater advantage of choosing a puncture point with Hasson trocars at the region with larger ascites as well as smaller puncture points of 1.0 to $1.2 \mathrm{~cm}$ diameter for catheters. Both B-mode
A

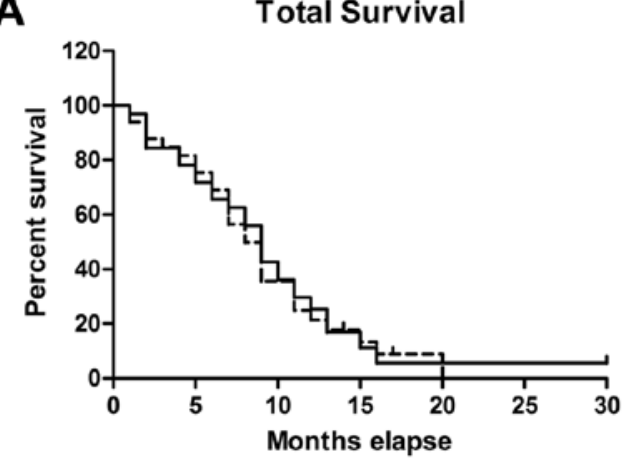

C

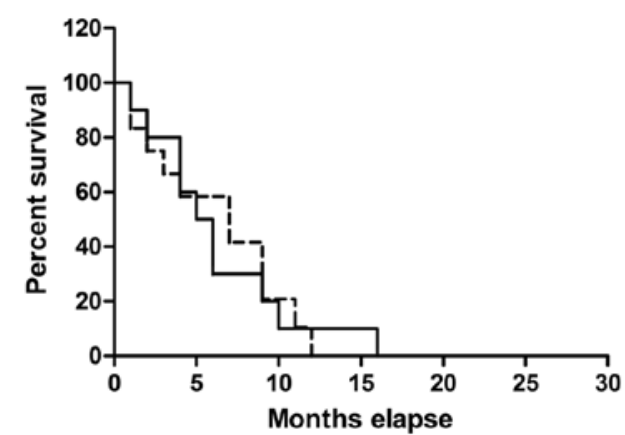

B Survival of $\mathrm{OC}$ patients

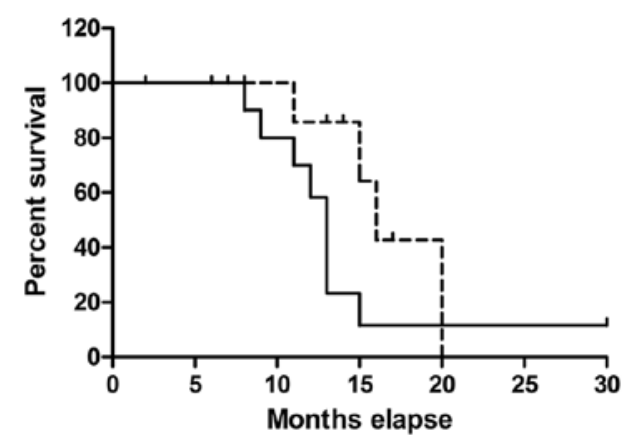

D Survival of CRC patients

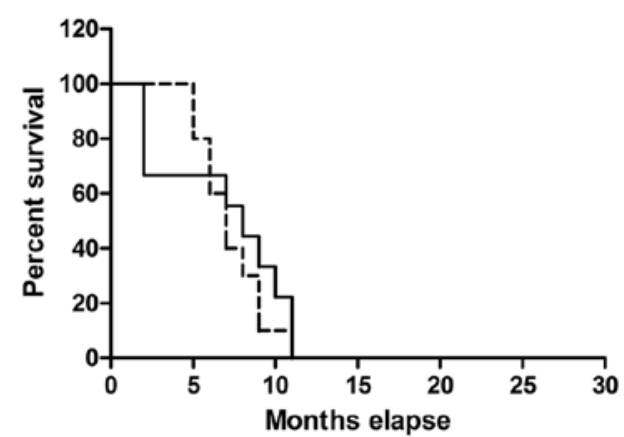

Figure 8. Comparison of survival curves of patients received B ultrasound guided and laparoscope assisted CHIPC. Curves of solid line represent survival of patients treated with ultrasound guided CHIPC, while dashed line mean the laparoscope assisted CHIPC. 
ultrasound guided and laparoscope assisted CHIPC were not only efficient in improving the patients' QOF, but also obtained equivalent efficacy in prolonging the survival time of patients $(\mathrm{p}=0.83)$ (Fig. 8A). Therefore, since 2008, this treatment method has become a standard treatment strategy for patients with ovarian carcinoma with massive ascites in our institution. In this study, we achieved an ORR of $93.75 \%$ for the ultrasound guided treatment of ascites symptoms with an improved median survival period of 9 months (from 2 to 30 months), compared to $93.90 \%$ ORR and 8-month survival time (from 2 to 20 months) of patients who had received laparoscope assisted CHIPC ( $>>0.05)$ (Table III). As to patients of different cancers, the survival time was not different either $(p=0.54,0.92$ and 0.12) for CRC, GC and OC patients treated with B ultrasound guided and laparoscope assisted CHIPC, respectively (Fig. 8B-D). There were fewer complications observed during sessions of CHIPC guided by ultrasound. Our clinical results demonstrated that B ultrasound-guided CHIPC is a safe, effective and feasible approach for patients suffering from a large mount of ascites with lesser complications.

We also found that, with the treatment of ascites, the prognosis seems to be closely associated with the origins of cancers. Patients with ovarian, colorectal and gastric cancers, respectively, have the best, better and moderate prognosis demonstrated by the length of survival period and improved QOF. This might suggest that the differences in prognosis among these patients could be related to the dissimilarities in: i) efficacy of the anticancer drugs used, ii) reactivity of the therapeutic reagents to the cancer tissues, and iii) the molecular and/or pathophysiological nature of tumors. Further investigation on the underlying mechanism is warranted.

Admittedly, laparoscopy has an advantage of detecting occult peritoneal carcinomatosis over B ultrasound, CT, MRI or PET-CT for evaluation of the stage of peritoneal malignant tumor in order to avoid any unnecessary surgery $(1,13,15,18,19,28)$. Therefore, for those patients with unknown primary lesion status of peritoneal cancer without surgical operation, especially with ovarian cancer, an exploratory laparotomy should be performed as soon as possible after the CHIPC together with new adjuvant chemotherapy for a better prognosis. Another issue worth considering during laparoscopic surgery is port-site seeding/ metastasis. Unfortunately, the ultrasound guided CHIPC failed to minimize the rate of port-site seeding (Table III), although this may not due to the manner of placing catheters.

In conclusion, we demonstrated a novel approach of using $\mathrm{B}$ ultrasound guided CHIPC in the treatment of malignant ascites with satisfactory outcomes. The approach took full advantage of MIS, and improved the patient's quality of life and prolong survival time. It could be potentially applied in other clinical applications.

\section{Acknowledgements}

This study was supported by The Funds for Breakthroughs in Key Areas of Guangdong and Hong Kong Project (no. 2006Z1E6041) and Guangdong Provincial Science and Technology Program Project Funds (no. 2009A030301013). The study protocol was approved by Cancer Hospital of Guangzhou Medical College (Guangzhou 510095, China).

\section{References}

1. Patriti A, Cavazzoni E, Graziosi L, et al: Successful palliation of malignant ascites from peritoneal mesothelioma by laparoscopic intraperitoneal hyperthermic chemotherapy. Surg Laparosc Endosc Percutan Tech 18: 426-428, 2008.

2. Becker G, Galandi D and Blum HE: Malignant ascites: systematic review and guideline for treatment. Eur J Cancer 42: 589-597, 2006.

3. Woopen $\mathrm{H}$ and Sehouli J: Current and future options in the treatment of malignant ascites in ovarian cancer. Anticancer Res 29: 3353-3359, 2009.

4. Aarts F, Hendriks T, Boerman OC, Koppe MJ, Oyen WJ and Bleichrodt RP: A comparison between radioimmunotherapy and hyperthermic intraperitoneal chemotherapy for the treatment of peritoneal carcinomatosis of colonic origin in rats. Ann Surg Oncol 14: 3274-3282, 2007

5. Kusamura S, Younan R, Baratti D, et al: Cytoreductive surgery followed by intraperitoneal hyperthermic perfusion: analysis of morbidity and mortality in 209 peritoneal surface malignancies treated with closed abdomen technique. Cancer 106: 1144-1153, 2006.

6. Levine EA, Stewart JH IV, Russell GB, Geisinger KR, Loggie BL and Shen P: Cytoreductive surgery and intraperitoneal hyperthermic chemotherapy for peritoneal surface malignancy: experience with 501 procedures. J Am Coll Surg 204: 943-953, 2007.

7. Spratt JS, Adcock RA, Muskovin M, Sherrill W and McKeown J: Clinical delivery system for intraperitoneal hyperthermic chemotherapy. Cancer Res 40: 256-260, 1980.

8. Zanon C, Bortolini M, Chiappino I, et al: Cytoreductive surgery combined with intraperitoneal chemohyperthermia for the treatment of advanced colon cancer. World J Surg 30: 2025-2032, 2006.

9. Al-Shammaa HA, Li Y and Yonemura Y: Current status and future strategies of cytoreductive surgery plus intraperitoneal hyperthermic chemotherapy for peritoneal carcinomatosis. World J Gastroenterol 14: 1159-1166, 2008.

10. Benoit L, Cheynel N, Ortega-Deballon P, Giacomo GD, Chauffert B and Rat P: Closed hyperthermic intraperitoneal chemotherapy with open abdomen: a novel technique to reduce exposure of the surgical team to chemotherapy drugs. Ann Surg Oncol 15: 542-546, 2008.

11. Huh JW, Kim YJ and Kim HR: Complete peritonectomy and intraperitoneal chemotherapy for recurrent rectal cancer with peritoneal metastasis. World J Gastroenterol 15: 756-757, 2009.

12. Spratt JS, Adcock RA, Sherrill W and Travathen S: Hyperthermic peritoneal perfusion system in canines. Cancer Res 40: 253-255, 1980.

13. Ba MC, Cui SZ, Lin SQ, et al: Chemotherapy with laparoscopeassisted continuous circulatory hyperthermic intraperitoneal perfusion for malignant ascites. World J Gastroenterol 16: 1901-1907, 2010

14. Facchiano E, Scaringi S, Kianmanesh R, et al: Laparoscopic hyperthermic intraperitoneal chemotherapy (HIPEC) for the treatment of malignant ascites secondary to unresectable peritoneal carcinomatosis from advanced gastric cancer. Eur J Surg Oncol 34: 154-158, 2008

15. Ferron G, Gesson-Paute A, Classe JM and Querleu D: Feasibility of laparoscopic peritonectomy followed by intra-peritoneal chemohyperthermia: an experimental study. Gynecol Oncol 99: 358-361, 2005.

16. Garofalo A, Valle M, Garcia J and Sugarbaker PH: Laparoscopic intraperitoneal hyperthermic chemotherapy for palliation of debilitating malignant ascites. Eur J Surg Oncol 32: 682-685, 2006.

17. Gesson-Paute A, Ferron G, Thomas F, de Lara EC, Chatelut E and Querleu D: Pharmacokinetics of oxaliplatin during open versus laparoscopically assisted heated intraoperative intraperitoneal chemotherapy (HIPEC): an experimental study. Ann Surg Oncol 15: 339-344, 2008.

18. Di Giorgio A, Naticchioni E, Biacchi D, et al: Cytoreductive surgery (peritonectomy procedures) combined with hyperthermic intraperitoneal chemotherapy (HIPEC) in the treatment of diffuse peritoneal carcinomatosis from ovarian cancer. Cancer 113: 315-325, 2008.

19. Elias D, Lefevre JH, Chevalier J, et al: Complete cytoreductive surgery plus intraperitoneal chemohyperthermia with oxaliplatin for peritoneal carcinomatosis of colorectal origin. J Clin Oncol 27: 681-685, 2009. 
20. Fujimoto S, Shrestha RD, Kokubun M, et al: Intraperitoneal hyperthermic perfusion combined with surgery effective for gastric cancer patients with peritoneal seeding. Ann Surg 208: 36-41, 1988.

21. Helm CW, Bristow RE, Kusamura S, Baratti D and Deraco M Hyperthermic intraperitoneal chemotherapy with and without cytoreductive surgery for epithelial ovarian cancer. J Surg Oncol 98: 283-290, 2008 .

22. Scaringi S, Kianmanesh R, Sabate JM, et al: Advanced gastric cancer with or without peritoneal carcinomatosis treated with hyperthermic intraperitoneal chemotherapy: a single western center experience. Eur J Surg Oncol 34: 1246-1252, 2008.

23. Shido A, Ohmura S, Yamamoto K, Kobayashi T, Fujimura T and Yonemura Y: Does hyperthermia induce peritoneal damage in continuous hyperthermic peritoneal perfusion? World J Surg 24: $507-511,2000$
24. Inadomi J, Cello JP and Koch J: Ultrasonographic determination of ascitic volume. Hepatology 24: 549-551, 1996.

25. Ozkan O, Akinci D, Gocmen R, Cil B, Ozmen M and Akhan O Percutaneous placement of peritoneal port-catheter in patients with malignant ascites. Cardiovasc Intervent Radiol 30: 232-236, 2007.

26. Kaushik N, Khalid A, Brody D and McGrath K: EUS-guided paracentesis for the diagnosis of malignant ascites. Gastrointest Endosc 64: 908-913, 2006.

27. Nguyen PT and Chang KJ: EUS in the detection of ascites and EUS-guided paracentesis. Gastrointest Endosc 54: 336-339, 2001.

28. Knutsen A, Sielaff TD, Greeno E and Tuttle TM: Staged laparoscopic infusion of hyperthermic intraperitoneal chemotherapy after cytoreductive surgery. J Gastrointest Surg 10: 1038-1043, 2006. 